Cahiers d'études africaines

\title{
Sorcellerie et délivrance dans les pentecôtismes
} africains

Sandra Fancello

\section{OpenEdition}

1 Journals

Édition électronique

URL : https://journals.openedition.org/etudesafricaines/10382

DOI : 10.4000/etudesafricaines.10382

ISSN : 1777-5353

Éditeur

Éditions de l'EHESS

\section{Édition imprimée}

Date de publication : 7 avril 2008

Pagination : 161-183

ISBN : 978-2-7132-2141-5

ISSN : 0008-0055

\section{Référence électronique}

Sandra Fancello, "Sorcellerie et délivrance dans les pentecôtismes africains », Cahiers d'études africaines [En ligne], 189-190 | 2008, mis en ligne le 08 avril 2011, consulté le 21 septembre 2021. URL http://journals.openedition.org/etudesafricaines/10382 ; DOl : https://doi.org/10.4000/ etudesafricaines.10382 


\title{
Sandra Fancello
}

\section{Sorcellerie et délivrance dans les pentecôtismes africains}

\begin{abstract}
«Pasteur,
Je vous prie de bien vouloir intercéder tous les jours jusqu'à la fin de l'année pour la délivrance de ma famille de la malédiction de mort. La santé de ma mère, tout ce qui bouge dans son corps, tout ce qui est intime, que Satan a placé dans son corps provenant de la sorcellerie et des mauvais esprits, [...] la délivrance des membres de la famille de l'esprit de la mort et du programme de la sorcellerie. »
\end{abstract}

Williamsville, Abidjan, Côte-d'Ivoire, 2002.

Comme le soulignait Evans-Pritchard (1972), chez les Azandé, et plus globalement en Afrique, la sorcellerie fait partie de l'ordinaire et n'a rien de mystérieux. Quels que soient les milieux, le discours sur la sorcellerie s'impose comme une réalité quotidienne de la vie sociale et des rapports humains, y compris dans le milieu urbain des sociétés africaines contemporaines, souvent en étroite relation avec «le village », considéré comme le foyer de la sorcellerie. Si le champ de l'imaginaire sorcellaire s'amplifie en milieu urbain, la famille et les proches demeurent traditionnellement considérés comme la source principale du pouvoir sorcier. La perception de la sorcellerie comme composante de la modernité urbaine africaine (Adam 2006 ; Ashforth 2005 ; Geschiere 2006 ; ter Haar 2007a), s'accompagne d'un autre constat: l'imaginaire sorcellaire semble traverser, voire transcender, les univers religieux. En témoigne le concept d' "insécurité spirituelle» ( "spiritual insecurity») associé à celui de «forces invisibles » ( invisible forces ») à partir desquels Adam Ashforth (2005) tente d'appréhender, dans le contexte sud-africain, la réalité sociale quotidienne d'un monde «peuplé de sorciers ». Ce concept hybride, qui rappelle l'expression de « forces occultes » utilisée par Peter Geshiere (1995), renvoie à la notion de «guerre spirituelle» (《spiritual warfare») dans le contexte chrétien. «L'insécurité spirituelle» ne se réduit pas à la sorcellerie traditionnelle, 
elle englobe, un peu à la manière des catégories missionnaires, les fétiches, les esprits païens, les sorciers, les démons et même le sida. La protection, voire la lutte, contre ces «forces invisibles » entretient un vaste marché de la guérison, manne des guérisseurs traditionnels ainsi que des Églises indépendantes, prophétiques et pentecôtistes. C'est aussi parce qu'elle participe pleinement de la «modernité africaine » que la sorcellerie accompagne l'explosion des pentecôtismes indigènes et que les catégories, autant que les frontières, de ces univers a priori distincts se déplacent. Pour Joseph Tonda (2000 : 48) : "Cette "modernité" de la sorcellerie remet un peu en cause la différence culturaliste instituée entre le dieu chrétien et le génie sorcier du paganisme. » La contribution active du pentecôtisme au phénomène de la sorcellerie est une composante majeure du succès de ce mouvement religieux en Afrique.

Le succès des pratiques de délivrance accompagne l'émergence des Ministères de délivrance et des camps de prière ${ }^{1}$ en Afrique depuis le début des années 1990. Au Ghana, l'attraction des Ministères de délivrance surpasse celle des shrines anti-sorcellerie et des Spiritual Churches. Cette pratique repose sur une vision dichotomique du monde perçu comme le terrain d'affrontement de la puissance divine contre les forces du Mal. La délivrance est étroitement associée à la guérison divine par le biais de la lutte contre les génies, esprits et démons considérés comme responsables des maux physiques et « spirituels » ainsi que de la «maladie », même si cette notion est centrée sur une série limitée de maux. Cette perception du rapport à la maladie, aux autres et au monde, se nourrit d'une série de représentations «persécutives » (Ortigues 1966: 225) à partir desquelles le fidèle se pense en proie aux puissances maléfiques dans tous les instants de sa vie. La délivrance se présente alors comme une libération de la souffrance et du Mal qui passe par l'exorcisme, l'éradication. La diabolisation des esprits païens, ancêtres et génies protecteurs, est le principal ressort de l'expansion des pentecôtismes africains. Comme l'observe Adam Ashforth (2005 : 312) à partir de l'Afrique du Sud : «Ancestors are also under increasing assault from the varieties of Pentecostalism that are spreading rapidly throughout Africa. »

Cet article s'appuie d'abord sur une série d'enquêtes menées au sein de la Church of Pentecost quasi simultanément dans trois pays : le Burkina Faso, la Côte-d'Ivoire et le Ghana depuis $2001^{2}$. Au Ghana, comme en Côted'Ivoire, la délivrance est une pratique courante que l'on peut observer soit

1. Les expressions pour les désigner sont nombreuses : Birgit MEYER (1999) utilise les appellations healing centre ou healing station, tandis que LARBI (2001) utilise celles de healing camps, prayer camps ou prayer centres et Mensa Otabil (ICGC) celle de Solution centre.

2. Dans le cadre de l'École des hautes études en sciences sociales, de l'unité de recherche 107 «Constructions identitaires et mondialisation » de l'IRD, ainsi que du programme « Réseaux transnationaux et nouveaux acteurs religieux en Afrique de l'Ouest », de l'Institut français de recherche en Afrique (IFRA) d'Ibadan (Nigeria). 
en tant que séquence intégrée au culte hebdomadaire, ou lors de veillées, soit dans l'espace des «camps de prière », qui sont des structures indépendantes associées à l'Église. En Côte-d'Ivoire, l'apparition des camps de prière peut être perçue dans la continuité historique des «villages thérapeutiques » conçus par les «prophètes guérisseurs » comme Albert Atcho et la communauté de Gregbo (Piault 1975; Augé 1990). L’influence des évangélistes américains et leurs témoignages de guérison personnelle sont également à l'origine de l'émergence des quelques grandes figures nigérianes et ghanéennes qui n'ont cessé de générer des vocations de «prophètes-guérisseurs » («healing prophets») dans leur pays et les pays voisins. Ce dynamisme créatif a produit plusieurs générations de leaders pentecôtistes ghanéens, mais aussi ivoiriens et burkinabè, de sorte que même les Églises les plus « classiques » sont amenées à prendre en compte ce constant renouvellement. Qu'ils aient une surface sociale internationale ou qu'ils soient des leaders « en leur pays », les « croyants-guérisseurs », «prophètes » ou «prophétesses », ces nouvelles figures hors normes et indociles, illustrent les rapports ambigus que les Églises pentecôtistes entretiennent avec la sorcellerie.

Le pentecôtisme ghanéen est aussi connu pour ses camps de guérison et de délivrance (Larbi 2001), des institutions thérapeutiques qui font appel à des techniques d'exorcisme et à une relecture des catégories de la sorcellerie. Les postures des Ministères de délivrance sont très différentes de celles des Églises pentecôtistes dites «classiques » qui n'acceptent pas la possibilité qu'un converti puisse être possédé par des démons. La Church of Pentecost, comme d'autres Églises de sa génération (Assemblies of God, Christ Apostolic Church, Apostolic Church, qui étaient encore les Églises pentecôtistes majeures jusque dans les années 1970) n'encourage pas les Ministères de délivrance, considérant que le baptême de l'Esprit apporte par lui-même salut, guérison et délivrance (Onyinah 2002 : 115) ${ }^{3}$. Nous verrons cependant comment, face à la frilosité des Églises pentecôtistes «classiques », les camps de prière et les Ministères de délivrance, sous la direction de leaders atypiques, intègrent dans la pratique de la délivrance des techniques du corps et de nouvelles représentations de la sorcellerie inspirées de la littérature évangélique américaine, dont les presses africaines se font le relais. Ces guérisseurs aux pouvoirs multiples s'inscrivent dans des réseaux transnationaux en marge de l'orthodoxie de l'Église à laquelle ils sont pourtant associés et disposent d'une popularité qui rivalise avec l'autorité pastorale. Dans ces structures marginales, le double jeu du pentecôtisme vis-à-vis de la sorcellerie africaine est encore plus perceptible. Il est même l'un des plus puissants attraits des centres de prières.

3. Directeur des Missions internationales de 1991 à 1996, l'Apôtre Opoku Onyinah est membre du Conseil exécutif international et le recteur du collège biblique de l'Église depuis 2002. Il est donc au centre de l'instance qui tranche sur la politique globale de la Church of Pentecost et de celle qui la diffuse. 


\section{Pasteurs contre féticheurs : une histoire ancienne}

L'histoire des pentecôtismes africains est marquée très tôt par la lutte contre la sorcellerie qui est l'un des ressorts de l'évangélisation. Parmi les précurseurs du pentecôtisme en Afrique de l'Ouest, le prophète W. W. Harris, parcourant la Côte-d'Ivoire et le Ghana dès le début du $\mathrm{Xx}^{\mathrm{e}}$ siècle, convertit, baptise, incite les villageois à renoncer à la sorcellerie et à brûler leurs fétiches. Plus tard, les pasteurs s'engagent à leur tour dans la lutte contre la sorcellerie comme préalable à la conversion. Dans le duel pasteurs/féticheurs qui s'engageait alors, la puissance du mal n'est pas simplement supprimée, elle est « inversée » ( turned around ») c'est-à-dire « retournée » contre son agent qui est «chassé » et finalement soumis à une puissance supérieure (Leonard 1989). Le face-à-face pasteurs/féticheurs se traduit donc par un rapport de forces et illustre l'ambivalence originelle de la puissance divine perçue comme une force magique supérieure à celle des sorciers mais relevant de la même nature. Comme le fait remarquer Adam Ashforth (2005: 181) concernant l'Afrique du Sud : "Conversions to Christianity, that is to say, which the missionaries proclaimed as the result of their earthly labors empowered by their Christian God, were often seen by the locals as a product of sorcery or other mystical forces. » Ce premier malentendu de la rencontre missionnaire marque l'histoire des christianismes africains.

Dans leur guerre spirituelle et par le discours de la «recrudescence» de la sorcellerie, les Églises pentecôtistes alimentent cet imaginaire sorcellaire, voire le réactivent et l'intègrent, tout en prétendant lutter contre. La Church of Pentecost du Ghana, dont la naissance même est liée au débat sur la guérison divine ${ }^{4}$, est traversée par les courants néo-pentecôtistes d'aujourd'hui et son histoire récente est jalonnée par une série de débats portant sur la délivrance, sa pratique, ses techniques et son encadrement institutionnel. Le statut de ces fidèles pas ordinaires, «croyants-guérisseurs » ou simples «visionnaires », est perçu de manière ambivalente par les Églises, comme l'Église de Pentecôte ou les Assemblées de Dieu, qui ont opté pour des structures pyramidales. Les dirigeants considèrent avec méfiance la réputation charismatique d'un «croyant-guérisseur » puisque son charisme lui permet en quelque sorte de court-circuiter les modes de promotion hiérarchique. Les fidèles leur accordent volontiers le titre de «prophète » ou plus simplement de «guérisseur » et s'accordent à leur reconnaitre un pouvoir supérieur à celui des «fonctionnaires » de l'Église, précisément du fait qu'ils sont en contact direct avec Dieu ou le Saint-Esprit tandis que les pasteurs obéissent à un ordre hiérarchique et sont soumis aux pesanteurs de l'orthodoxie.

4. Cette Église est en partie le résultat de l'affiliation du jeune ghanéen Peter Anim avec l'Apostolic Church (Bradford) auprès de laquelle Anim sollicita l'envoi d'un missionnaire en Gold Coast. La collaboration entre Anim et McKeown prit fin à la suite du départ d'Anim qui, influencé par le fondamentalisme américain, condamnait le recours à la médecine au profit de la prière de guérison (imposition des mains). Pour une analyse détaillée voir FANCELLO (2006). 
La «profession de foi anti-sorcière » (Tonda 2000 : 50) des pasteurs et des convertis intègre, non sans ambivalence, les représentations traditionnelles africaines qu'elle reformule en faisant de la figure du diable l'interface entre les deux systèmes de croyance : «Embrayeur de communication entre le système sorcellaire traditionnel et une démonologie moderne dominée par les rapports de force, le Réveil du Diable participe surtout à la réactivation d'un schéma persécutif du Mal qui encourage, sur un mode euphémisé, l'accusation de l'autre proche aussi bien que la diabolisation de l'étranger, à commencer par l'inconverti. C'est à ce prix que le Diable devient l'agent double de la conversion » (Mary 1998). Pour Birgit Meyer (1998b), qui parle depuis le Ghana, la délivrance, synonyme de « rupture totale avec le passé », comprend deux dimensions : la première correspond à la délivrance du passé personnel (immediate past), la seconde à une libération de la «malédiction ancestrale » (ancestral past), une conception «persécutive » du Mal qui se transmet à travers les générations. Ainsi, les tensions familiales étant attribuées à la sorcellerie ou aux esprits ancestraux (Akan spirits, siis buudu $u^{5}$, Mami Wata), la rupture avec le passé implique parfois concrètement la rupture des liens familiaux. Comme le résume la formule de Birgit Meyer (1998a: 75) : «Le Démon œuvre à travers les liens du sang; le Dieu chrétien les rompt. »C'est la plasticité de ces catégories qui permet de les maintenir aussi efficaces dans le registre pentecôtiste que dans leur usage initial. Les biographies et les récits de vocation des croyants-guérisseurs sont à l'image de cette plasticité, mêlant à la fois les ressources des «guérisseurs » traditionnels, de leur initiation à l'islam ou de l'héritage catholique, et celle de la puissance de l'Esprit. L'ambivalence des discours tient aux multiples sources qui sont mobilisées, mêlées et bientôt confondues au point, parfois, de brouiller les pistes qui ont amené à ces discours complexes et parfois contradictoires.

\section{Sorcellerie et démonologie : les "plans de l'ennemi »}

L'influence du mouvement néo-pentecôtiste pétri de littérature américaine a fortement modifié le paysage religieux ouest-africain depuis les années 1990. Les pays anglophones comme le Ghana et le Nigeria jouent un rôle majeur dans la diffusion des nouveaux concepts religieux tels que celui de la «malédiction ancestrale », selon lequel la conversion ne garantit pas la protection contre le pouvoir maléfique des ancêtres. Certaines Églises se sont approprié ces théories, regroupées sous le terme de witchdemonology,

5. Si dans la langue möré, les pasteurs utilisent l'expression siis buudu, en français ils utilisent plutôt l'expression « esprits de la famille » au sens de famille "élargie » qui n'est pas le strict équivalent du lignage mais qui renvoie à une catégorie «globalisée » de la sorcellerie dont la langue française est, avec l'anglais, l'un des vecteurs. 
qui comprend la lutte contre la sorcellerie, les démons et la malédiction ancestrale. Le terme witchdemonology est une combinaison d'un terme emprunté à la culture traditionnelle africaine, revisitée par l'anthropologie anglo-saxonne (Evans-Pritchard), désignant la sorcellerie proprement dite, witchcraft - et non sorcery qui désigne la magie - , et du terme occidental demonology. La witchdemonology est le produit d'une hybridation, au sens de Bakhtin ${ }^{6}$, des notions occidentales et africaines relatives à la sorcellerie (Onyinah 2002 : 118). Les termes «sorcier» (witch) et sorcellerie (witchcraft) sont utilisés comme des synonymes de démon et de mauvais esprit (evil spirit). Ainsi, les prêtres traditionnels (priests) ${ }^{7}$ sont assimilés à des sorciers. Cette théorie, inspirée entre autres par les américains Kenneth Hagin et Peter Wagner (et sa notion de spiritual warfare), implique un lien direct entre les mauvais esprits (anges déchus), les démons et les catégories de génies africains (abosom pour les Ashanti) qui peuplent la brousse (forêts, rivières, arbres) et l'imaginaire akan traditionnel. Cette vision du monde est également véhiculée en Afrique par les ouvrages de Derek Prince (1998: 148) pour qui la sorcellerie est «la cinquième colonne dans la société et dans l'Église ». La possibilité qu'un chrétien, converti, ayant reçu le baptême d'eau mais aussi le baptême de l'esprit, reste victime de la malédiction ancestrale qui pèse sur sa famille, est au cœur du sujet : «Demons might be in a person before one became a Christian. Moreover, demons might enter a person after he/she has become a Christian » (cité par Onyinah 2002 : 116). L'évangéliste américaine Rebecca Brown (1986 : 233) va plus loin, en suggérant que des démons «satanistes » infiltrent régulièrement et détruisent des églises chrétiennes. Le sorcier c'est toujours l'autre, le proche.

Le concept pentecôtiste de la «malédiction ancestrale » implique que la conséquence des péchés (ou considérés comme tels) commis par les « anciens » retombe sur la lignée descendante. La thèse est relayée par certains pasteurs pentecôtistes ghanéens comme Owusu Tabiri ${ }^{8}$. Elle a pour complément pratique la nécessité de la délivrance associée à la rupture des liens du sang (breaking ou obubu en twi) qui passe par l'identitfication des démons ancestraux : « rooting the Devil» (Tabiri 2004 : 63). Les effets de la malédiction ancestrale sur une personne se traduisent par des maladies chroniques, des accidents psychopathologiques (mental breakdown), des

6. «In historical, organic hybrids it is not only two languages but also two sociolinguistic (thus organic) world views that are mixed with each other; but in such situations, the mixture remains mute and opaque, never making use of conscious contrast and oppositions » (BAKHTIN 2002: 360).

7. L'usage du terme anglais priest par les Ashanti renvoie en fait à l'okòmfó (pl. akòmfó), féticheur (charmer, sorcerer) lit. "celui qui est possédé », composé avec akóm, état de celui qui est possédé ou fetish-dance. Il communique avec les abosom et interprète leur message.

8. Dissident de la Church of Pentecost (1995) et fondateur du Bethel Prayer Ministry International. 
excès émotifs, des allergies, des pertes financières répétées, une succession de morts non naturelles (telles que les accidents ou les suicides) dans l'entourage de la personne, des divorces répétés ou des troubles du comportement tels de la mauvaise humeur, des excès de colère ou, à l'inverse, une réserve excessive. Les traits de caractère et les aléas de la vie quotidienne sont ainsi interprétés comme les effets de la malédiction ancestrale : ce sont les «plans de l'ennemi », les «plans de sabotage» de Satan pour éprouver les chrétiens et les détourner de leur «destinée prophétique », c'est-à-dire $\mathrm{du}$ «plan de Dieu» pour chaque individu.

Dans ses aspects pratiques, la délivrance consiste à identifier les esprits démoniaques ou à les reconnaître en fonction de symptômes décrits par la plupart des manuels de délivrance d'inspiration pentecôtiste ou charismatique tels qu'en produisent les centres d'éditions africains, souvent ivoiriens ou nigérians (Adekoya 1997). L'exorcisme proprement dit (la fuite des démons) passe par l'imposition des mains et parfois une lutte au corps à corps avec le "possédé », associées à un usage pragmatique d'extraits bibliques, choisis en fonction de la «maladie», comme l'illustre, dans le contexte catholique charismatique, le célèbre traité de Meinrad P. Hebga ${ }^{9}$ (1986 : 54-65, 2006).

\section{La sorcellerie en questions}

Ces techniques de lutte contre la sorcellerie se retrouvent au Burkina Faso, voisin du Ghana, associées à des innovations qui empruntent aux méthodes du marketing et visent cette fois un public urbain et scolarisé. À Ouagadougou, le Centre d'intercession, de délivrance et d'évangélisation (CIDE) a mis au point un questionnaire destiné à identifier les « mauvais esprits » en mettant davantage l'accent sur la consultation individuelle comme préalable à la délivrance collective qui aura lieu plus tard, lors du culte hebdomadaire. La consultation, qui se déroule souvent dans la cour du Centre, passe par un entretien avec le leader qui propose au «malade» de répondre à une série de questions afin de cerner la cause de son mal. Les questions portent sur l'environnement familial de la personne ( «y a-t-il un membre de votre famille, arrière-grand-père ou arrière-grand-mère, tante ou oncle, qui fut prêtre ou prêtresse des fétiches, ou un roi, reine, y a-t-il un imam, un guerrier ? »); les antécédents de santé («avez-vous une maladie héréditaire ?»); les appartenances religieuses antérieures («avez-vous déjà participé à des rites ? », «avez-vous déjà participé à des danses culturelles, danses des

9. Meinrad P. Hebga est un prêtre camerounais du courant charismatique, formé à la théologie à Rome, mais aussi à la philosophie (Sorbonne) ainsi qu'à la psychologie générale et pathologique. Son ouvrage, déjà classique, qui se présente comme un manuel de lutte contre la sorcellerie et participe à la production de méthodes de diagnostic en matière de sorcellerie, veut offrir, selon la présentation de l'auteur, «des moyens spirituels de lutte contre les forces du mal». 
masques, [...] ou autre forme d'idoles, bonne aventure, magie blanche, horoscope, astrologie ? [...] à des groupes transcendantaux tels que la RoseCroix, Eckankar? » $)^{10}$.

Le patronyme d'une personne peut être considéré comme une source de «blocage» pour le converti : "Parce que ici, presque chaque famille est liée à une idole, explique le pasteur: les Sawadogo, les Zabré, les Ouedraogo, etc. On adore quelque chose, comme le cheval, et ça joue sur la vie chrétienne. Il y a aussi un animal que la famille évite à cause d'un dieu. » Les patronymes mossi se rapportent en effet à des personnages historiques de la dynastie des Ouedraogo, symbolisent des pouvoirs magiques $(\text { sawadogo })^{11}$ ou un héritage ancestral: ainsi zabré désigne les guerriers mais peut aussi être associé à un trait de caractère ${ }^{12}$. Les porteurs de ces patronymes peuvent être victimes de cet héritage ancestral perçu comme malfaisant, vecteur de la malédiction ancestrale. La nomination de l'enfant fait partie de cet héritage : «T'a t-on fait qu'un membre de ta famille s'est réincarné ?»Le prénom donné à l'enfant peut être considéré comme une source de «blocage » lorsqu'il est donné en hommage à un ancêtre ${ }^{13}$ : «Ici c'est comme ça, ils disent que c'est le grand-père qui est revenu et quand la personne veut se convertir, il y a des problèmes, parce que ce sont des démons de nécromancie, ce n'est pas la personne qui est revenue, mais chaque année ils donnent un poulet, donc nous prions pour briser tout ça et pour que la personne ne croie plus à ces choses-là », explique le pasteur. Ces types de «blocages » sont attribués à une "maladie spirituelle », qui affecte directement ou indirectement la relation à Dieu et peuvent expliquer qu'un chrétien, même une fois converti, soit poursuivi et tourmenté par de mauvais esprits. La nécromancie (évocation des morts pour apprendre d'eux l'avenir) est un travestissement du pouvoir que les Mossi accordent aux rêves dans lesquels un ancêtre bienveillant peut apparaître afin de les prévenir d'un danger imminent ( Voyez-vous des choses dans vos rêves qui se réalisent ? »), parfois assimilé à « l'esprit de mort», qui fait voir des défunts en rêve. Les pentecôtistes assimilant le «monde invisible», où se donnent à voir les ancêtres — tantôt bienveillants, tantôt persécuteurs —, doubles ${ }^{14}$

10. Eckankar est un groupe spirituel transcendantal fondé en 1965 par l'Américain Paul Twitchell. Il a son siège à Chanhassen (Minnesota). Eckankar est présent à Ouagadougou.

11. De sa-wadgho, nuage. Associés aux nyônôsé, les «enfants de la terre " ("têngbiisi »), ils ont le pouvoir de se transformer en vent et de déclencher tempêtes et tourbillons (silmandé).

12. De zab, dispute, querelle, comme dans la phrase a nôga zabré : il aime la dispute. Par extension, combat, bataille, guerre. Bao Wênnaam zabré : chercher, provoquer la colère de Dieu.

13. Notons que le terme "réincarné » utilisé par les pasteurs est inadéquat car l'ancêtre dont on transmet le nom, le grand-père par exemple, est davantage un esprit protecteur que la réincarnation à travers le nouveau-né.

14. Siiga chez les Mossi, le double, qui est aussi la force vitale, est à la fois sujet et objet de sorcellerie, tandis que chez les Akan, c'est le sunsum qui est l'agent de la sorcellerie mais c'est le kra, force vitale, que les sorciers attaquent. Notons 
et sorciers, à une vaste supercherie démoniaque, interprètent comme la manifestation de démons toute apparition en rêve, voire les rêves eux mêmes : "Voyez-vous des choses que les autres ne voient pas ? Entendezvous des voix ? "Ces facultés attestent de la proximité de démons dans l'environnement de la personne qui entend des voix (ce sont des démons qui lui parlent) mais qui peut aussi les voir, sans se douter qu'elle est seule à voir ce que les autres ne voient pas.

Le rapport à l'argent joue également un rôle important dans l'interprétation symptomatologique que constitue l'entretien par questionnaire : « Avezvous souvent des surprises d'argent ? »; être dépensier ou à l'inverse gagner de l'argent de manière inattendue: «si tu as 1000 CFA et tu vois que ça devient $6000 \mathrm{CFA}$, il y a quelque chose qui ne va pas », explique le pasteur. La perte d'objets ou à l'inverse la découverte d'objets inconnus : «perdezvous des objets ? », « avez-vous des biens qui ne vous appartiennent pas ?». «C'est quand tu vois dans tes habits ou tes bijoux quelque chose que tu n'as jamais acheté, ça arrive ici », commente le pasteur. La perte d'objets est une étourderie attribuée aux démons qui parasitent l'esprit de la personne mais il peut s'agir d'un vol destiné à diriger une attaque en sorcellerie, de même que la découverte d'un objet inconnu, placé à dessein au domicile de la personne. L'objet suspect est considéré comme le vecteur de la puissance maléfique. Enfin, certains traits de caractère ou de la personnalité sont également attribués à l'entremise de mauvais esprits : « Êtes-vous méchant, têtu, coléreux, paresseux ? » Le fait par exemple «d'agiter les doigts de manière mécanique, indépendante de la volonté », ajoute le pasteur, est considéré comme un signe nerveux de dépendance envers un démon. Caractérologie et démonologie vont de pair.

Les appartenances religieuses antérieures du converti font aussi l'objet de l'interrogatoire. L'islam est considéré comme une «fausse doctrine » qui égare l'individu, notamment par son rapport de compromis avec les pratiques traditionnelles et la sorcellerie. La seule présence d'un imam dans la famille du converti peut être considérée comme la source de ses «blocages ». Le caractère confrérique de la Rose-Croix et de la Franc-maçonnerie alimente l'imaginaire sorcelleraire au point que, en Afrique de l'Ouest comme en Afrique centrale, leurs membres peuvent être « accusés de détenir les magies internationales les plus redoutables [...] on leur attribue pratiques incestueuses, homosexualité, meurtres rituels et d'autres pratiques symbolisant la sorcellerie la plus disruptive » (Tonda 2002 : 51). De même, les groupes « transcendantaux» sont au minimum perçus comme des groupes sectaires dangereux. Au côté de ces mouvements notons l'allusion du pasteur aux expériences de la «bonne aventure », de la «magie blanche », voire de

que dans les catégories pentecôtistes, et respectivement dans les langues möré et twi, l'expression qui désigne le Saint-Esprit est composée des termes Siigsôngo et Onyame Sumsum, faisant des deux entités ambivalentes une composante de l'Esprit divin. 
l'astrologie ou de l'horoscope, qui sont classées dans la catégorie des «idoles de superstitions ». Le résultat de ce questionnaire est censé permettre de diagnostiquer l'origine du mal. Que le «malade»soit un fidèle régulier ou un non converti, le pasteur lui propose de se présenter au temple lors du prochain culte afin de recevoir une prière de guérison. La médiation du questionnaire introduit une mutation importante par rapport aux procédures divinatoires traditionnelles. Le pasteur qui engage le dialogue oral dans un entretien par questionnaire se réfère à l'autorité de l'écriture et d'un savoir accumulé dans les traités et les manuels pratiques de lutte contre la sorcellerie.

\section{Delivremoi.com}

Le recours au questionnaire est une pratique répandue dans d'autres Ministères de délivrance tels que Gem Kakou Ministries, ministère chrétien fondé par le prophète ivoirien Gem Kakou, qui propose sur son site Internet un test en ligne afin de savoir si l'on est ensorcelé ${ }^{15}$. Les questions-test portent souvent sur les mêmes thèmes. Des thèmes qui n'ont rien de « spirituel », mais qui pointent les problèmes de la vie ordinaire : la maladie ; l'environnement familial : «vous avez des génies protecteurs dans votre vie ou votre famille »; les traits de caractère, qui renvoient, on l'a dit, à la « malédiction ancestrale »: " vous êtes coléreux; vous oubliez beaucoup trop vite »; le rapport à l'argent : "vous dépensez plus que vous ne gagnez »; la réussite professionnelle : «vous échouez dans tout ce que vous entreprenez »; les problèmes conjugaux : «vous vous sentez mieux en dehors de chez vous »; ou encore l'image de soi : "vous avez une image négative de vous », associée à l'état psychologique : "désintérêt dans vos entreprises », qui renvoie aux rapports sociaux : " on vous tient des promesses qu'on ne respecte pas ; l'on vous emprunte mais l'on ne vous rembourse pas; vous êtes sujet à la tromperie ». Les aléas de la vie quotidienne sont aussi considérés comme des indices : " vous êtes régulièrement victimes d'accidents; pertes répétées et mystérieuses de vos objets; vous arrivez régulièrement en retard à vos rendez-vous importants »; ainsi que le décès de proches : "pertes répétées et mystérieuses de membres de votre famille ». Une réponse par l'affirmative à une ou plusieurs de ces questions permet d'identifier l'esprit importun, voire plusieurs, responsable(s) des «maladies », «blocages » ou « malédictions » qui pèsent sur la personne. À l'issue du questionnaire en ligne sur le site Gem Kakou Ministries, quelles que soient les réponses cochées par l'internaute, le résultat qui s'affiche va dans le même sens : «Si vous êtes concerné par trois éléments de la liste du formulaire, vous subissez une

15. «Y a-t-il une malédiction dans ta vie ? Test en ligne », <//www.gemkakou.org> qui propose un lien avec le site <www.delivremoi.com>, également géré par GKM. 
influence démoniaque, si vous êtes concerné par sept éléments de la liste du formulaire vous devez réagir urgemment. »Ainsi, par le biais de cette innovation technique, un chrétien ivoirien, à Abidjan — ou à Paris — peut à tout moment se connecter et recevoir un «diagnostic » en ligne ${ }^{16}$, même s'il s'agit davantage d'une méthode de prosélytisme que d'un diagnostic individuel.

La délivrance telle qu'elle se présente aujourd'hui, individuelle ou collective, se met en scène par une lutte au corps à corps entre le leader, vecteur de la puissance divine, et le fidèle (qui est souvent une femme ${ }^{17}$ ) dont l'agitation est interprétée comme la manifestation des démons qui le «tiennent». Qu'elles soient intégrées au culte hebdomadaire ou à un rassemblement public (type croisade), les séances de délivrance sont de puissants attraits aussi bien pour les fidèles réguliers que pour les non convertis en quête de guérison ou de miracles. Le discours de diabolisation contribue à évacuer la notion de responsabilité personnelle au profit des démons et mauvais esprits que les pasteurs dénoncent publiquement et incitent à se manifester dans le temple («montre-toi maintenant ! ») afin de les combattre et de les chasser («plie bagages, au nom de Jésus ! ), faisant ainsi l'économie de la confession. Ces appels répétés provoquent les premières crises de possession au sein même de l'assemblée dont les fidèles les plus agités sont tirés de la foule et déposés auprès du pasteur et de ses assistants qui entament une prière de délivrance, laquelle tourne rapidement à la lutte physique. Pratiquée aussi bien par des pasteurs que par des prophètes-guérisseurs, plus ou moins intégrés à l'orthodoxie, la délivrance repose sur une connaissance du mal qui peut être anticipée (prophètes) ou révélée (visionnaires) et porte aussi bien sur des maux «physiques» (parmi lesquels la stérilité des femmes, l'épilepsie ou la «folie » sont les plus fréquemment nommés) que « spirituels », qui se traduisent par des échecs économiques et sociaux (vie familiale, échecs professionnels). Cette perception du mal tend à dépasser la séparation entre guérison physique, corporelle et guérison psychique, « spirituelle » dans le traitement des « corps souffrants ».

Techniquement, la pratique de l'imposition des mains, inspirée de la Bible, s'est enrichie d'une large palette de techniques du corps plus ou moins brutales qui peuvent parfois mobiliser plusieurs personnes autour d'un «possédé ». Ainsi, «la délivrance relève moins d'un tribunal de la conscience que d'un combat total où le pasteur s'engage fortement et physiquement » (Mary 2001). Bien que très fréquente, la chute à terre est une manifestation physique de la délivrance plutôt controversée, de même que les cris, pleurs et vomissements (Fancello 2006). Tout d'abord parce que

16. Gem Kakou Ministries est implanté simultanément à Abidjan et Paris. Enregistré en février 2006 au Journal Officiel, il organise en juillet 2006 «l'École de la délivrance » à Paris, un cycle d'enseignements destiné à « briser les malédictions ».

17. Pour une étude sur le rapport spécifique des femmes à la délivrance, voir FANCELLO (2005). 
l'agitation extrême laisse penser que la délivrance passe nécessairement par une lutte acharnée entre le Saint-Esprit et les mauvais esprits, tandis que les pasteurs affirment que, par la prière et l'imposition des mains, le guérisseur délivre du mal «au nom de Jésus », lequel suffit à mettre en fuite les démons. La fonction performative du langage qui caractérise ce « discours de puissance », amplifie le pouvoir magique de guérison. Mais surtout, l'expulsion des mauvais esprits n'est assimilée à aucune expulsion réelle, d'aucune substance interne. Outre la violence des techniques du corps mobilisées, la délivrance s'accompagne d'un discours aux métaphores guerrières. Cette « guerre spirituelle» est un combat contre le Mal, et les pasteurs sont des « soldats de Dieu » («Warriors of God», «Prayer Warriors ») qui pratiquent la « chasse aux sorcières ». Cependant, là encore, «l'autre » agresseur ne se réduit pas aux sorciers mais englobe «l'autre» (les musulmans, les païens, l'étranger, le migrant, l'opposant politique, les amis, la famille). Toutes les sphères de la vie familiale, sociale et politique peuvent être sources de suspicion.

Au Burkina Faso, le «prophète » Emmanuel Sawadogo, dont l'itinéraire est passé par la Côte-d'Ivoire, fait partie des « croyants-guérisseurs » ${ }^{18}$ les plus populaires du pays. Sa technique de délivrance plutôt «musclée » lui vaut le surnom de «boxeur de la foi » (Laurent 2003). Dans le registre de l'oralité, le prophète Emmanuel invite les fidèles à une confession préalable à la délivrance. Ici, il faut «dire son problème » pour ouvrir la voie à la guérison. Mais c'est le prophète qui donne son diagnostic quasi invariable : «C'est des démons » (« ya zina »). Comme beaucoup de « croyantsguérisseurs », Emmanuel s’inspire des théories de la «malédiction ancestrale » («Si tes parents étaient coléreux, ça se répète directement sur toi ») mais en conclusion d'une séance de délivrance, il ne manque pas d'évoquer aussi le recours au pardon comme voie d'apaisement des conflits. Le pardon est une condition nécessaire au salut et à la délivrance : « $\mathrm{Si}$ vous avez de la colère contre quelqu'un, il faut pardonner d'ici à mercredi ( $k w \hat{e}$ soba sugri), de manière à ce que l'on puisse prier pour vous. Si votre cœur a la paix, Dieu exauce vos prières. » L'impératif de pardon consiste à l'accorder, sans demande préalable et sans condition, à la personne envers laquelle on nourrit un désir de vengeance ou une rancune tenace, et vise à rétablir «l'entente» ( «wum taaba»), une forme de pacification des relations sociales par le compromis. La « rancune » est une notion associée à la sorcellerie et elle occupe une place centrale au cœur du processus de guérison

18. L'expression est de P.-J. Laurent (1999, 2003 : 312) qui définit le « croyantguérisseur » comme « un fidèle ayant reçu de l'Esprit saint le don de guérison, auquel s'ajoute fréquemment, selon les cas, le don de discernement, le don de parler en langues [...], les dons de puissance, d'interprétation et de prophétie » et trouve «plus approprié de les nommer croyant (wënnam no-rèsa) (l'interprète de Dieu) ou wënnam tum-tuumda (celui qui fait le travail de Dieu)». 
d'un « prophète-guérisseur » ivoirien comme Sebim Odjo : «Nkpiti, la "rancune", cette perversion intime des relations sociales les plus intenses [...] c'est le facteur socio-psychologique sans l'existence duquel il n'y aurait ni sorcellerie, ni malédiction d'aucune sorte » (Augé 1990 : 41). Mais l'accusation directe n'est pas de mise dans les Églises. La délivrance a pour but de libérer les individus de l'entremise des démons qui les tourmentent sans faire peser sur eux de responsabilité directe. Lorsque Emmanuel ordonne « il faut pardonner », il veut rappeler que, dans un contexte chrétien cette fois, l'exorcisme des «démons de la colère » passe par l'acte de pardon, c'est-à-dire la libération de la rancune et le renoncement au cycle de la violence réciproque.

\section{Pasteurs ou prophètes guérisseurs}

En principe, les pasteurs de la Church of Pentecost, qui sont des « fonctionnaires de l'Église », s'en tiennent à un charisme de fonction et n'exercent pas de Ministères de guérison, lesquels reposent le plus souvent sur le charisme personnel du leader, perçu comme peu orthodoxe dans une Église extrêmement hiérarchisée. L'ambivalence des Églises et des pasteurs visà-vis de la sorcellerie et des esprits païens est variable selon les régions d'Afrique. En Afrique centrale (Congo, Gabon), les frontières entre christianisme et sorcellerie semblent plus floues qu'en Afrique de l'Ouest, au point que : «Au Congo, l'exorciste officiel de l'Église catholique, l'abbé Isidore Malonga, consulté pour ses pratiques de "guérison divine", est dit appartenir à une famille de grand nganga » (Tonda 2000 : 55). Certes, l'Église catholique est souvent perçue comme davantage compromise avec les pratiques magiques traditionnelles que les Églises pentecôtistes qui prônent la rupture totale avec la société traditionnelle, ses croyances et ses pouvoirs. Cependant, ces mêmes Églises tolèrent des structures telles que les camps de prière, de guérison et de délivrance, souvent fondés et dirigés par des personnages atypiques dont le charisme est inversement proportionnel à leur statut hiérarchique. Tandis que l'Église ne leur reconnaît rarement plus que le statut de diacre (ou diaconesse) ou au mieux d'ancien (un statut fermé aux femmes), ces leaders passent pour des personnalités charismatiques dont la réputation internationale est bien supérieure à celle des pasteurs et apôtres de l’Église. De plus, la priorité donnée à la quête de guérison donne à la délivrance une dimension clientéliste qui pervertit, aux yeux de l'Église, le rôle des responsables de camps.

Si la structure des camps de prière est moins répandue au Burkina Faso que dans les pays côtiers, on recense cependant plusieurs initiatives associées à l'Église de Pentecôte ou, plus souvent, à l'Église des Assemblées de Dieu. Plusieurs personnages, guérisseurs plus ou moins populaires, occupent aujourd'hui la scène burkinabè tels que le «vieux Ahmado » dans la région 
de Kombissiri (Église de Pentecôte), Emmanuel ${ }^{19}$ (AD), ou parmi les femmes, «Maman Suzanne » (AD), accueillent des «malades » de toutes confessions. Dans la capitale, le Centre international d'évangélisation de Mamadou Karambiri, l'un des personnages les plus populaires au Burkina Faso, reçoit les malades pour des prières de guérison. Les «croyants-guérisseurs » réputés pour leur «pouvoir de guérison » et parfois élevés au rang de «prophètes » par les fidèles, sont des vecteurs de la puissance divine, comme l'explique André Soubeiga (1999 : 129) : «La prière, au nom de Jésus, procède d'un transfert de pouvoir, le Christ déléguant son pouvoir et sa capacité de guérison à quiconque le sollicite. » Contrairement aux «travailleurs de Dieu » décrits par Joseph Tonda (2002: 158), dans le contexte du Congo et du Gabon et «qui sont des lettrés d'un niveau moyen largement au-dessus des nganga [les guérisseurs traditionnels] », les « croyants-guérisseurs » que nous avons rencontrés au Burkina Faso, en Côte-d'Ivoire et au Ghana, sont en général d'un niveau d'instruction minimal, souvent illettrés, et à la différence des «travailleurs de Dieu», ce ne sont jamais des pasteurs ${ }^{20}$. Ces «croyants-guérisseurs » sont plus proches de ce que Joseph Tonda (2002: 99) appelle des « identités valorisées de convertis », engagés dans des « carrières de spécialistes de la guérison ».

La formation du Centre d'intercession et de délivrance (CIDE) au Burkina Faso à la fin des années 1980 participe du dynamisme créatif des Églises africaines en matière de lutte contre la sorcellerie. Le CIDE se forma à partir d'une cellule de prière dans l'un des temples des Assemblées de Dieu du quartier de la Patte d'Oie (secteur 15). La réputation grandissante de la cellule de prière aboutit à la création du Centre d'intercession, de délivrance et d'évangélisation (CIDE) au milieu des années 1980. Dans sa déclaration d'association du 4 juin 1992, le CIDE précise qu'il «condamne la sorcellerie » et fait de la délivrance son activité principale, recevant des fidèles de toutes confessions. Mais bientôt, l'indépendance du CIDE par rapport à l'Église des Assemblées de Dieu et l'aspect clientéliste de la fréquentation du Centre - la conversion n'étant pas une condition de la guérison l'Église tente de réintégrer le Centre dans ses activités : «Normalement, les $\mathrm{AD}$ ne reconnaissent pas la délivrance, mais quand ils ont vu comment on faisait, ils ont vu que c'était pas mauvais. Et ils ont accepté ça », raconte le pasteur en charge de l'assemblée du quartier Ouaga 2000 où est installé le temple actuel du CIDE.

19. P.-J. LauRent $(1999,2003)$, plus familier de l'Église des Assemblées de Dieu au Burkina Faso, a consacré une partie de ses analyses sur les « croyants-guérisseurs » à Emmanuel aujourd'hui devenu un personnage public.

20. À l'exception de Mamadou Karambiri, fondateur du Centre international d'évangélisation, ou de Yao Bio en Côte-d'Ivoire, dissident de l'Église de Pentecôte, ou encore de Owusu Tabiri au Ghana, également dissident de la Church of Pentecost et fondateur de sa propre Église. Ces «pasteurs » trouvent davantage leur légitimité en tant que «fondateurs d'Église » qu'au sein d'une hiérarchie ecclésiale. 
Ces prophètes guérisseurs et guérisseuses, inscrits dans un réseau régional, apparaissent comme des personnages d'autant plus marginaux qu'ils ne se pensent pas eux-mêmes comme étroitement liés à l'Église à laquelle ils sont pourtant associés, ou affiliés, plus ou moins formellement. À l'image des prophètes nigérians tels que Babamuboni, Egunjobi ou Moses Orimolade décrits par John Peel (1968) et qui prêchaient de village en village, sans véritable affiliation aux Églises établies, invoquant le seul pouvoir de la prière. Cette indépendance relative vis-à-vis des Églises locales est un trait commun des «guérisseurs » qu'illustraient par exemple la posture du « prophète » Sebim Odjo vis-à-vis de l'Église méthodiste (Augé 1990), ou Harris en son temps (Haliburton 1984). L'échantillon des fidèles du «vieux » Ahmado, qui n'affiche pas la dénomination «Église de Pentecôte », est en fait constitué de villageois de Pellé et des quelques kilomètres alentour. Certains, comme Ahmado, Emmanuel, ou parmi les femmes, Sister Kate au Ghana, Maman Haggar en Côte-d'Ivoire ou Maman Suzanne et Maman Doris au Burkina Faso, s'affirment avant tout comme des fidèles ayant reçu un pouvoir divin dont les voies court-circuitent la hiérarchie de l'Église au sein de laquelle ils n'avaient souvent aucun statut. Pour donner libre cours à leur don de guérison, vis-à-vis duquel les Églises telles que l'Église de Pentecôte ou les Assemblées de Dieu ont une attitude mitigée, certains leaders « révélés » ont choisi de prendre leur indépendance, tel Owusu Tabiri, fondateur du Bether Prayer Camp, qui quitta la Church of Pentecost du Ghana pour fonder la Bethel Prayer Church en 1995. Cette rupture alimenta un long débat au sein de la Church of Pentecost sur l'importance de la délivrance dans la lutte contre la sorcellerie (Baidoo 1995).

\section{« Exorcisms Are a Part of our Culture»}

L'idée selon laquelle la sorcellerie a considérablement augmentée durant ces dernières décennies est entretenue dans le milieu pentecôtiste par la plupart des ouvrages de la littérature évangélique essentiellement produite par les évangélistes américains (Prince 1998; Joyner ${ }^{21} 2004$ : 106). Cette perception est en partie due au fait que dans le milieu pentecôtiste le champ de la sorcellerie s'étend à toutes les formes d'interactions sociales et ne concerne pas spécifiquement le continent africain : «When we try to use emotional pressure to manipulate others, it is a basic form of witchcraft [...]. Many of the manipulative tactics promoted as sales techniques in marketing are basic forms of witchcraft »(Joyner 2004 : 108).

21. L'Américain Rick Joyner est fondateur des Morning Star Publications and Ministries. 
SÉRIE D’OUVRAGES CHRÉTIENS, AMÉRICAINS ET AFRICAINS PORTANT SUR LA SORCELLERIE

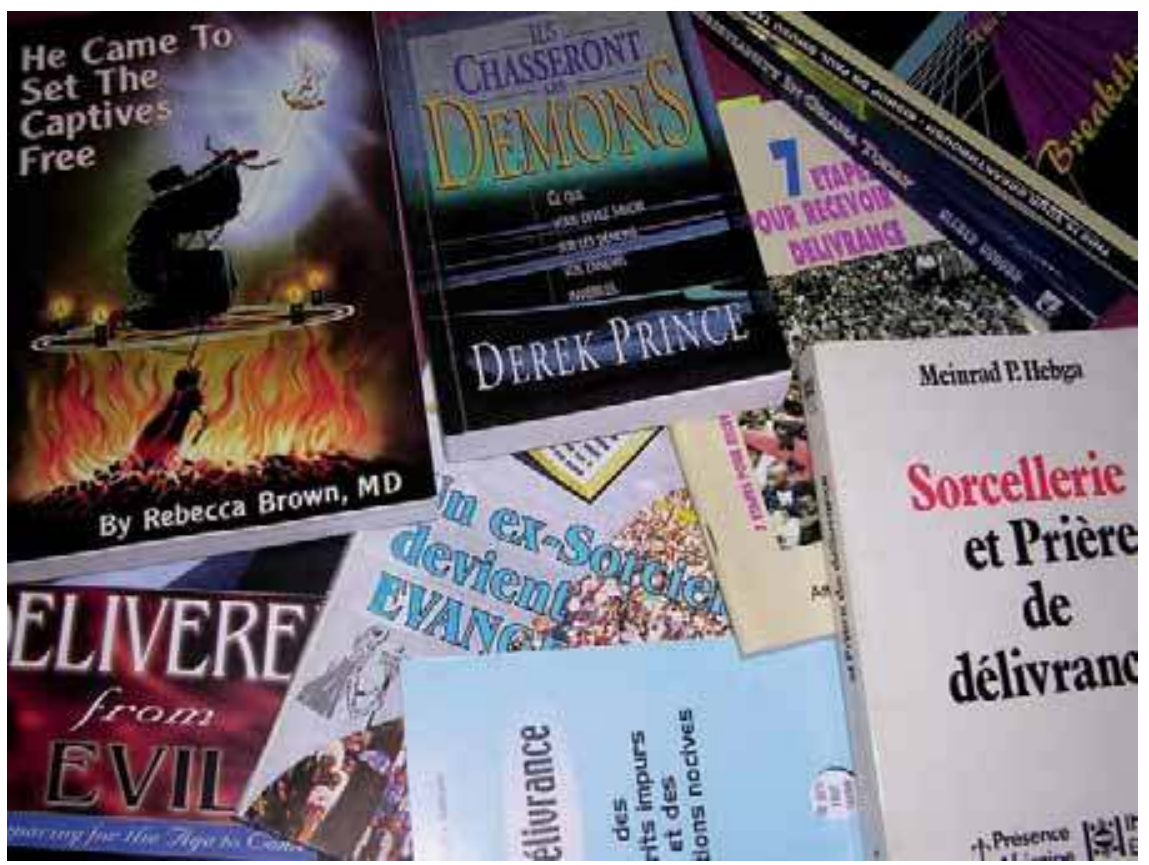

La globalisation du champ de la sorcellerie aboutit à la production de nouvelles catégories d'esprits à caractère psychologique qui se déclinent à volonté : «esprit du doute» proche du Spirit of confusion (Joyner 2004 : 106), « esprit du chômage », " esprit du souci », « esprit de l'ennemi », et bien sur « esprit de sorcellerie ». Les entreprises prophétiques de « rationalisation » du diagnostic se nourrissent des sciences « occultes » qui sont, au même titre que les imaginaires initiatiques de la Rose-Croix ou la Francmaçonnerie, des produits ésotériques qui excitent l'imaginaire africain et réveillent les cosmogonies locales. Attribuer des pouvoirs aux astres, en faire des marqueurs d'identité, transformer le signe de naissance en un « double », solidaire de traits de caractère et définissant la personnalité d'un individu. La lecture d'une réussite matérielle ou professionnelle en termes astrologiques définissant les atouts d'une personne placée sous ce signe assimile ces jeux ésotériques à des productions symboliques du pouvoir blanc que Joseph Tonda (2002: 50) appelle le «Génie Blanc». La perception africaine du Génie blanc fait de l'évolution matérielle et technologique des sociétés occidentales le produit de ses sciences occultes : "Les produits du génie scientifique que sont les voitures, les avions, les appareils électroménagers, les moyens de télécommunications, bref, toutes ces choses "extraordinaires" (au sens aussi de choses fascinantes) qui suscitent envie, frustrations, jalousies, haines familiales ou de classe, désirs de mort de l'autre 
possédant, etc. Pour la pensée "indigène" ordinaire, ces objets traduisent la nature extraordinaire de leur producteur, le "Blanc" [...] ce n'est pas par hasard si des histoires de sorcellerie font de ces choses des moyens par excellence de l'action des sorciers. »

L'implantation d'Églises «africaines » sur le sol européen participe de cette globalisation de l'imaginaire sorcellaire. Certains aspects « indigènes » de leur «culture pentecôtiste » (transe, délivrance, guérison miraculeuse) contribuent à stigmatiser, non sans amalgame, l'irruption de ces «Églises noires » dans le paysage religieux européen. Des affaires récentes comme celle qui a éclaté à Londres à propos de la mort d'un enfant lors d'une séance de délivrance dans une Église «congolaise», remettent ces sujets sur la scène médiatique et font porter le soupçon sur l'ensemble des Églises pentecôtistes africaines assimilées aux «sectes». Ces pratiques, qualifiées de $«$ satanisme $»^{22}$ et de $«$ magie noire ${ }^{23}$ par la presse, ont fait l'objet d'un rapport de la police britannique, restitué par la $\mathrm{BBC}$ en juin $2005^{24}$, dans une affaire concernant l'arrestation de deux femmes accusées de tortures à l'égard d'une fillette originaire d'Angola. Accusée de sorcellerie, la fillette fut lacérée au couteau, battue et affamée, en guise de «traitement » («illtreatment »), des poivrons du Chili furent frottés dans ses yeux jusqu'à ce qu'elle avoue. Notons que, dans ces circonstances dramatiques qui aboutissent parfois à la mort d'un enfant, l'enjeu, c'est toujours l'aveu et non pas la mise à mort du sorcier. Au domicile de la femme qui s'était chargée de faire entrer la fillette en Angleterre en la faisant passer pour sa fille, la police trouve des documents relatifs au péché (sin), au diable (devil) et à la sorcellerie (witchcraft), ainsi qu'un journal personnel, écrit en lingala : « There was indeed a prophesy she has ndoki [the lingala word for witchcraf] $»^{25}$. Cette histoire se fait l'écho d'une affaire similaire, le cas d'une fillette originaire de Côte-d'Ivoire, accusée d'être possédée, et décédée des suites des blessures infligées au cours d'une séance de délivrance. Ces affaires ont attiré l'attention des autorités britanniques sur les Églises africaines, notamment le Ministère de combat spirituel ${ }^{26}$, un Ministère de délivrance fondé à Kinshasa en $1993^{27}$. Le pasteur congolais Modeste Muyulu,

22. <www.top chrétien.org>, juillet 2005 .

23. Jeune Afrique, AFP/Intelligent.com, 16 juin 2005.

24. "Girl tortured "for being a witch" », BBC News, 9 may 2005.

25. "Witch" child cruelty trio guilty », BBC News, 3 June 2005. Le terme ndoki désigne le pouvoir sorcier dans la langue lingala qui couvre une partie de l'Afrique centrale.

26. Le nom de cette Église, qui n'apparaît pas dans les articles diffusés par la BBC en juin 2005, fut dévoilé au cours d'un documentaire intitulé L'enfant du diable (Allemagne, Royaume Uni, Congo, 2006, 43 mn), WDR, réalisé par Sacha Mirzoeff, diffusé le mardi 19 septembre 2006, sur Arte. Au cours de ce documentaire, Richard Hoskins, africaniste expert auprès de la police britannique, suit la trace d'un jeune garçon, né en Angleterre de parents congolais, envoyé à Kinshasa par sa famille afin d'être exorcisé.

27. Fondé par Elisabeth Olangi, le Ministère de combat spirituel est associé à la Fondation Olangi-Wosho qui a son siège à Kinshasa. 
président de la Pentecostal Church of the French Christian Community Bethel (CCFB), affirme : «Exorcisms are a part of our culture. » Dans cette Église, composée d'une centaine de membres originaires d'Angola et du Congo, le pasteur justifie les pratiques de délivrance par la menace que représentent certains individus «ensorcelés »: «There are people who are used by the devil to bring a curse or bad luck to other people's lives, even to kill them $»^{28}$. Les enquêtes menées par la police britannique, avec l'aide d'un « expert africaniste », font état de la disparition de centaines d'enfants alimentant un trafic d'enfants africains. À travers ces «affaires de sorcellerie » l'on assiste à la transnationalisation du phénomène de l'accusation de sorcellerie, comme le suggèrent les travaux produits à partir d'autres terrains européens (ter Haar 2007 : 6, 103).

Ces violences de l'aveu ne sont pas sans évoquer le phénomène des « enfants-sorciers » observé au Congo (Kinshasa) depuis plusieurs années, où les Églises du Réveil, ou certains prophètes qui s'en réclament, jouent un rôle non négligeable. La mise en cause des enfants est un phénomène qui s'impose à la réalité quotidienne du milieu urbain congolais (De Boeck 2000: 34). Tenu pour responsable d'une maladie, d'un décès ou d'un divorce, «l'enfant du diable » est accusé de sorcellerie et devient la victime expiatoire du malheur de sa famille. S'il arrive que certains soient «tués par des membres de la famille ou des voisins », la plupart des enfants accusés sont «simplement rejetés et reniés » $\left(\right.$ ibid. : 39) ${ }^{29}$. Dans ce contexte, certaines Églises pentecôtistes accueillent les enfants-sorciers, mais le diagnostic est souvent effectué par les pasteurs eux-mêmes, parfois à la demande de la famille. Les prophètes ou pasteurs nganga contribuent à faire exister le phénomène de la sorcellerie enfantine : «La contribution des Églises joue un rôle crucial dans l'incessante production et la mise en évidence croissante de la figure du sorcier dans l'imaginaire collectif » (ibid. : 40). Dans leur lutte anti-sorcière, ces Églises s'exposent aux procès engagés par des institutions civiles (éventuellement laïques ou sociales) qui pointent de telles pratiques et récupèrent les enfants incriminés ${ }^{30}$. Le phénomène des enfantssorciers illustre ainsi le paradoxe d'Églises prises elles-mêmes dans le piège de l'accusation de sorcellerie alors qu'elles prétendent lutter contre les sorciers. Entre le sorcier et le contre-sorcier, l'inversion des places est une constante.

28. «Exorcisms are part of our culture», BBC News, Friday 3 June, 2005.

29. En juin 2001, de présumés sorciers de cinq ou six ans ont été massacrés dans la région d'Aru, dans le nord-est du pays, à la frontière de l'Ouganda, voir Libération du 18 juillet 2001 .

30. Voir à ce sujet le reportage de Élisabeth Burdot et Édith van Hove, La nouvelle stratégie du Diable, RTBF, 2004. 


\section{Le cercle du pasteur et du sorcier}

La représentation de la sorcellerie comme composante de la modernité africaine établit un lien fort entre les univers de croyances multiples qui travaillent les sociétés contemporaines. Le «combat spirituel» contre le Mal est devenu une catégorie générique, qui en fait une réalité quotidienne et le principe explicatif de la souffrance et du malheur. La circulation des pratiques d'exorcisme et des affaires de sorcellerie entre l'Afrique et l'Europe participe de cette globalisation de l'imaginaire selon lequel «le diable est partout et nulle part ».

Mais cette globalisation opère en même temps des changements significatifs sur le fond et sur la forme. Ainsi le recours au diagnostic, oral ou écrit, par la confession téléguidée ou le questionnaire plus ou moins directif, permet d'inscrire le démon dans l'examen systématique des petits maux du quotidien autant que dans l'univers de l'occulte, en pratiquant une sorte de psychologisation des esprits (Csordas 1997). Le recours au questionnaire est une pratique inspirée des manuels pratiques de délivrance - qui n'est pas sans rappeler les manuels de confession et les interrogatoires pénitentiels de l'évangélisation missionnaire — mais sa diffusion sur l'Internet apparaît comme une innovation qui contribue à transcender les frontières entre la coutume, le livre et le web, sans pour autant faire l'économie du passage obligé par une communauté de délivrance. Dans la plupart des Églises pentecôtistes, les accusations de sorcellerie désignent souvent un membre de la famille proche, l'oncle maternel, le mari jaloux ou la femme rivale, mais elles ne débouchent pas sur l'inversion de la violence ou le passage à l'acte. Néanmoins comme le souligne André Mary (1998) : «Toutes les lectures chrétiennes qui s'efforcent de donner une version acceptable de la sorcellerie comme facteur de régulation des conflits intra-familiaux, instrument d'éradication de la violence et de réintégration du sorcier dans la communauté, misent sur les vertus libératrices de l'aveu et sur l'apaisement du pardon mais passent sous silence les ressources curatives de la décharge d'agressivité sur un autre persécuteur. » Mais depuis peu, le phénomène des « enfants-sorciers » ou des «enfants du Diable » opère, dans un contexte urbain d'insécurité et d'infortune, une inversion inquiétante du soupçon et des règles du jeu de l'accusation qui ciblaient traditionnellement les vieux du village, voire les ancêtres disparus, les femmes étant toujours assignées à leur place de «sorcières ». Ainsi, la globalisation des catégories de la sorcellerie et de la délivrance amène à prendre en compte les formes de renouvellement de ces pratiques, entre autres, par le jeu des questionnaires qui viennent court-circuiter la confession, ou l'usage de l'Internet qui participe de la déterritorialisation des pratiques comme des esprits.

Centre d'études des mondes africains, CNRS, Paris. 


\section{BIBLIOGRAPHIE}

ADAM, M.

2006 «Nouvelles considérations dubitatives sur la théorie de la magie et de la sorcellerie en Afrique noire », L'Homme, 177-178 : 279-302.

Adekoya, A.

19977 étapes pour recevoir la délivrance, Abidjan, Éditions World Wide Jesus Crusade.

AshForth, A.

2005 Witchcraft, Violence, and Democracy in South Africa, Chicago, University of Chicago Press.

AugÉ, M.

1990 Nkpiti, la rancune et le prophète, Paris, Éditions de l'EHESS.

BAIDOO, S. K. (Apôtre)

1995 «What is Deliverance ?», Pentecost Fire, $104: 18-20$.

BAKHTin, M. M.

2002 [1981] The Dialogic Imagination, Austin, University of Texas Press.

BROWN, R.

1986 He Came To Set The Captives Free, New Kensington, Whitaker House.

CsORDAS, T. J.

1997 The Sacred Self : A Cultural Phenomenology of Charismatic Healing, Berkeley, University of California Press.

De Boeck, F.

2000 «Le "deuxième monde" et les "enfants-sorciers" en République démocratique du Congo », Politique africaine, 80 : 32-57.

Evans-Pritchard, E. E.

1972 [1937] Sorcellerie, oracles et magie chez les Asandé, Paris, Gallimard.

FANCELLO, S.

2003 «Les politiques identitaires d'une Église africaine transnationale: The Church of Pentecost (Ghana) », Cahiers d'Études africaines, XLIII (4), 172 : 857-881.

2005 «Pouvoirs et protections des femmes dans les Églises pentecôtistes africaines », Revista de Estudos da Religião (« Gender and Religion»), 3 : 78-98.

2006 Les aventuriers du pentecôtisme ghanéen. Nation, conversion et délivrance en Afrique de l'Ouest, Paris, IRD-Karthala.

GESCHIERE, P.

1995 Sorcellerie et politique, la viande des autres, Paris, Karthala.

2006 «The State, Witchcraft and the Limits of the Laws. Cameroon and South Africa », in E. DE Rosny (dir.), Justice et sorcellerie, Paris, Karthala-Presses de l'UCAC : 87-120. 
TER HAAR, G.

2007a «Introduction: The Evil Called Witchcraft», in G. TER HAAR (ed.), Imagining Evil. Witchcraft, Beliefs and Accusations in Contemporary Africa, Africa World Press, Inc. : 1-30.

2007b «Ghanaian Witchcraft Beliefs : A View form the Netherlands », in G. TER HAAR (ed.), op. cit. : 93-112.

HALiburton, M.-G.

1984 Le prophète Harris, Abidjan, Les nouvelles éditions africaines.

HeBGA, M.

1986 Sorcellerie et prière de délivrance, Paris, Présence Africaine; Abidjan, inades Éditions.

2006 « Approche et pratique pastorale », in E. DE RoSNY (dir.), op. cit. : 325-329.

JOYNER, R.

2004 Delivered from Evil, Shippensburg, Destiny Image Publishers, Inc.

LARBI, E. K.

2001 Pentecostalism. The Eddies of Ghanaian Christianity (Centre of Pentecostal and Charismatic Studies), sapc, series 1, Accra.

LAURENT, P.-J.

1999 «L'Église des Assemblées de Dieu au Burkina Faso, histoire, transitions et recompositions identitaires », Archives de Sciences sociales des Religions, 105 : 71-97.

2003 Les pentecôtistes du Burkina Faso. Mariage, pouvoir, guérison, Paris, IRDKarthala.

LEONARD, C.

1989 A Giant in Ghana. 3,000 churches in 50 years. The Story of James McKeown and The Church of Pentecost, Chichester, New Wine Press.

MARY, A.

1998 «La diabolisation du sorcier et le réveil de Satan», Religiologiques, Marges contemporaines de la religion, Montréal, Université du Québec : 53-77.

2001 «From One Syncretism to Another: Culture of Trance and Charisma of Deliverance ", Social Compass, Prophète, visionnaires et guérisseurs de l'Afrique subsaharienne contemporaine, 48 (3) : 315-331.

MEYer, B.

1992 " "If You Are a Devil, You Are a Witch and, if You Are a Witch You Are a Devil". The Integration of "Pagan" Ideas into the Conceptual Universe of Ewe Christians in Southern Ghana », Journal of Religion in Africa, XXII (2) : 98-131.

1998a «"Make a Complete Break with the Past". Memory and Post-colonial Modernity in Ghanaian Pentecostalist Discourse », Journal of Religion in Africa, XXVIII (3) : 316-349.

1998b «Les églises pentecôtistes africaines, Satan et la dissociation de "la tradition" », Anthropologie et sociétés, 22 (1): 63-83.

1999 Translating The Devil. Religion and Modernity among The Ewe in Ghana, Edinburgh, Edinburgh University Press. 
ONYINAH, O.

2002 «Deliverance as a Way of Confronting Witchcraft in Modern Africa: Ghana As a Case History », Asian Journal of Pentecostal Studies, 5 (1) : 107-134.

Ortigues, M.-C. \& Ortigues, E.

1966 Edipe africain, Paris, Plon.

PeEl, J. D. Y.

1968 Aladura: A Religious Movement Among the Yoruba, London, Oxford University Press.

Piault, C. (dir.)

1975 Prophétisme et thérapeutique: Albert Atcho et la communauté de Bregbo, Paris, Hermann.

PRINCE, D.

1998 Ils chasseront les Démons, Internet, DPMF <www.DPMF.net>.

Soubeiga, A.

1999 «Quête de guérison, conversion, évangélisation. Groupes charismatiques et Églises pentecôtistes face au Mal », in R. Otayek (dir.), Dieu dans la cité. Dynamiques religieuses en milieu urbain ouagalais, Bordeaux, CEAN : 111-128.

TABIRI, O.

2004 This is Your Breakthrough, Logos, Publishing House KIA ; Accra-Ghana.

TONDA, J.

2000 «Capital sorcier et travail de Dieu», Politique africaine, 79: 48-65.

2002 La guérison divine en Afrique centrale (Congo, Gabon), Paris, Karthala («Hommes et Sociétés »).

\section{RÉSUMÉ}

Le discours sur la sorcellerie s'impose comme une réalité quotidienne de la vie sociale et des rapports humains, y compris dans le milieu urbain des sociétés africaines contemporaines. Si le champ de l'imaginaire sorcellaire s'amplifie en milieu urbain, la famille et les proches demeurent traditionnellement considérés comme la source principale du pouvoir sorcier. La perception de la sorcellerie comme composante de la modernité urbaine africaine accompagne l'explosion des pentecôtismes indigènes. La contribution active du pentecôtisme au phénomène de la sorcellerie, par la diabolisation des esprits païens, ancêtres et génies protecteurs, entretient un vaste marché de la guérison, manne des guérisseurs traditionnels ainsi que des églises indépendantes, prophétiques et pentecôtistes. La délivrance se présente alors comme une libération de la souffrance et du mal qui passe par l'exorcisme, l'éradication. 


\section{ABSTRACT}

Sorcery and Deliverance in African Pentecostalisms. - The discourse on witchcraft is essential like a daily reality of the social life and human relationship, including in the urban environment of the contemporary African societies. If the field of witchcraft imaginary increase in urban environment, the family, always linked to the "village", remain traditionally regarded as the primary source of the witchcraft. The perception of witchcraft as component of African urban modernity societies goes with the explosion of indigenous Pentecostalism. The active contribution of the Pentecostalism to the witchcraft phenomenon, by the demonization of pagan spirits and ancestors, maintains a vast market of healing, manna of the traditional healers as well as independent, prophetic and Pentecostal Churches. Deliverance then appears like a liberation from suffering and Evil which goes through exorcism and eradication.

Mots-clés/Keywords : délivrance, démonologie, pentecôtisme, sorcellerie, violence/ deliverance, demonology, pentecostalism, witchcraft, violence. 